TMUP-HEL-9908

WU-HEP-99-4

June 1999

\title{
Solar Neutrinos and Leptonic CP Violation
}

\author{
Hisakazu Minakatain \\ Department of Physics, Tokyo Metropolitan University \\ 1-1 Minami-Osawa, Hachioji, Tokyo 192-0397, Japan, and \\ Research Center for Cosmic Neutrinos, Institute for Cosmic Ray Research, \\ University of Tokyo, Tanashi, Tokyo 188-8502, Japan \\ Shinji Watanabef \\ Department of Physics, Waseda University \\ 3-4-1 Okubo, Shinjuku-ku, Tokyo 169-8555, Japan
}

\begin{abstract}
We examine the possibility of detecting effects of leptonic $\mathrm{CP}$ violation by precise measurement of the solar neutrinos within the framework of standard electroweak theory minimally extended to include neutrino masses and mixing. We prove a "no-go theorem" which states that effects of $\mathrm{CP}$ violating phase disappear in the $\nu_{e}$ survival probability to the leading order in electroweak interaction. The effects due to the next-to-leading order correction is estimated to be extremely small, effectively closing the door to the possibility we intended to pursue.
\end{abstract}

\footnotetext{
†minakata@phys.metro-u.ac.jp

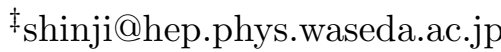


Exciting discovery of neutrino oscillation in atmospheric neutrino observation [1] strongly suggests that neutrinos are massive. Then, it is likely that the leptonic CKM [2] matrix, which is now called [3] as the Maki-Nakagawa-Sakata (MNS) [4] matrix, exists so that the nature admits $\mathrm{CP}$ violation in the lepton sector. The $\mathrm{CP}$ violation in the lepton sector has been the topics of interests because of various reasons; on the one hand it may inherit a key to understanding the lepton-quark correspondence, and on the other hand it may provide us with an intriguing mechanism for generating baryon asymmetry in our universe [5].

It is then important to explore the methods for measuring leptonic $\mathrm{CP}$ violating phase. Neutrino oscillation has been known to be one of the promising ways of measuring effects of $\mathrm{CP}$ violation [6]. Its effect may be detectable if the flavor mixing angles are not small. Recently, detailed investigations have been done to investigate how to measure $\mathrm{CP}$ violation in long-baseline neutrino oscillation experiments, with particular emphasis on how to discriminate matter effect contamination from the genuine effect of the Kobayashi-Maskawa phase [0 [1].

We consider in this paper an alternative possibility of measuring $\mathrm{CP}$ violation by precise measurement of the solar neutrino flux, assuming that neutrinos interact as dictated by the standard electroweak theory. Unfortunately, we will end up with the "no-go theorem". That is, we will show that the effects of $\mathrm{CP}$ violating phase vanish in any observables in solar neutrino experiments to leading order in the electroweak interaction. While the "theorem" is not quite a theorem because its validity is limited to leading order, it effectively closes the door to any practical attempts to measure $\mathrm{CP}$ violating effects in solar neutrino experiments because the higher order effects are so small. Or, in other word, if a single effect of $\mathrm{CP}$ violating phase is detected in a solar neutrino experiment, then it would imply the existence of the neutrino interactions beyond that of the standard model.

The goal of our argument in the first half of this paper is to show that the effect of $\mathrm{CP}$-violating phase vanishes to first order in electroweak interactions in the survival probability $P\left(\nu_{e} \rightarrow \nu_{e}\right)$. Notice that the solar neutrino observation is inherently a disappearance experiment; there is no way to detect appearance events in charged current interactions 
because the energy is well below the thresholds, and the neutral current interaction cannot distinguish between $\nu_{\mu}$ and $\nu_{\tau}$.

Toward the goal let us start by recalling the well known results for vacuum neutrino oscillation. There exists a general theorem that states that the measure for $\mathrm{CP}$ violation vanishes in the disappearance probability, i.e.

$$
P\left(\nu_{\alpha} \rightarrow \nu_{\alpha}\right)-P\left(\bar{\nu}_{\alpha} \rightarrow \bar{\nu}_{\alpha}\right)=0
$$

in vacuum. Hereafter, the Greek indices label the flavor eigenstates; $\alpha=e, \mu, \tau$ for threegenerations. This comes from the CPT invariance which implies that $P\left(\nu_{\alpha} \rightarrow \nu_{\beta}\right)=P\left(\overline{\nu_{\beta}} \rightarrow\right.$ $\left.\overline{\nu_{\alpha}}\right)$. The lepton flavor mixing is described by the mixing matrix $U_{\alpha i}$ that relates the flavor eigenstate $\nu_{\alpha}$ and mass eigenstate $\nu_{i}$ as $\nu_{\alpha}=U_{\alpha i} \nu_{i}$, where $\mathrm{i}$ is the index for specifying mass eigenstates.

In the framework of three flavor mixing the feature implied by the general theorem has a simple realization; the effect of $\mathrm{CP}$ violation shows up in the vacuum neutrino oscillation probabilities in the particular way as

$$
P\left(\nu_{\beta} \rightarrow \nu_{\alpha}\right)-P\left(\overline{\nu_{\beta}} \rightarrow \overline{\nu_{\alpha}}\right)=4 J_{\beta \alpha} \sin \left(\frac{\Delta m^{2} L}{2 E}\right),
$$

for $\alpha \neq \beta$ and $J_{\beta \alpha}$ stands for the leptonic Jarlskog factor [12],

$$
J_{\beta \alpha}=\operatorname{Im}\left[U_{\alpha 1} U_{\alpha 2}^{*} U_{\beta 1}^{*} U_{\beta 2}\right],
$$

the unique fermion rephasing invariant measure for $\mathrm{CP}$ violation. In fact, the $\mathrm{CP}$-violating piece in $P\left(\nu_{\beta} \rightarrow \nu_{\alpha}\right)$ is identical with that of $P\left(\bar{\nu}_{\beta} \rightarrow \bar{\nu}_{\alpha}\right)$ apart from the sign, and is given by a half of the right-hand-side of (2). By unitarity the disappearance probability can be written in terms of the appearance probabilities as $1-P\left(\nu_{e} \rightarrow \nu_{e}\right)=P\left(\nu_{e} \rightarrow \nu_{\mu}\right)+P\left(\nu_{e} \rightarrow \nu_{\tau}\right)$. The CP-violating pieces in the right-hand-side of this equation cancel owing to the cyclic property of $J_{\beta \alpha}$. This is all about how the $\mathrm{CP}$ violating effect vanishes in the survival probabilities $P\left(\nu_{\alpha} \rightarrow \nu_{\alpha}\right)$ in vacuum neutrino oscillation.

The situation changes completely when the matter effect [13] is taken into account. Since the matter is in general neither CPT nor CP symmetric there is no general argument 
which enforces that CP violation effect disappears in neutrino oscillation. Moreover, the CP violation which shows up in neutrino conversion probabilities contains both the fake matter and the genuine effects due to the Kobayashi-Maskawa phase. Therefore, it is difficult to make general statement on how $\mathrm{CP}$ violating effects come in.

To attack the problem we write down the evolution equation of three flavor neutrinos in matter which is valid to leading order in electroweak interaction:

$$
i \frac{d}{d x}\left[\begin{array}{c}
\nu_{e} \\
\nu_{\mu} \\
\nu_{\tau}
\end{array}\right]=\left\{U\left[\begin{array}{ccc}
m_{1}^{2} / 2 E & 0 & 0 \\
0 & m_{2}^{2} / 2 E & 0 \\
0 & 0 & m_{3}^{2} / 2 E
\end{array}\right] U^{+}+\left[\begin{array}{ccc}
a(x) & 0 & 0 \\
0 & 0 & 0 \\
0 & 0 & 0
\end{array}\right]\right\}\left[\begin{array}{c}
\nu_{e} \\
\nu_{\mu} \\
\nu_{\tau}
\end{array}\right]
$$

where $a(x)=\sqrt{2} G_{F} N_{e}(x)$ indicates the index of refraction with $G_{F}$ and $N_{e}(x)$ being the Fermi constant and the electron number density, respectively.7

We take a particular parametrization of the mixing matrix [14].

$$
U=e^{i \lambda_{7} \theta_{23}} \Gamma_{\delta} e^{i \lambda_{5} \theta_{13}} e^{i \lambda_{2} \theta_{12}}
$$

where $\lambda_{i}$ are $S U(3)$ Gell-Mann's matrix and $\Gamma$ contains the CP violating phase

$$
\Gamma_{\delta}=\left[\begin{array}{ccc}
1 & 0 & 0 \\
0 & 1 & 0 \\
0 & 0 & e^{i \delta}
\end{array}\right]
$$

We rewrite the evolution equation (4) in terms of the new basis defined [15] by

$$
\begin{aligned}
\tilde{\nu_{\alpha}} & =\left[e^{-i \lambda_{5} \theta_{13}} \Gamma^{-1} e^{-i \lambda_{7} \theta_{23}}\right]_{\alpha \beta} \nu_{\beta} . \\
& \equiv\left(T^{t}\right)_{\alpha \beta} \nu_{\beta}
\end{aligned}
$$

It reads

\footnotetext{
*We note, in passing, that the extra phases which appear for Majorana neutrinos do not give any effects in neutrino evolution in vacuum and in matter because they are multiplied from right to $U$ and therefore drop out in (雨).
} 


$$
i \frac{d}{d x}\left[\begin{array}{c}
\tilde{\nu_{e}} \\
\tilde{\nu_{\mu}} \\
\tilde{\nu_{\tau}}
\end{array}\right]=\left\{\frac{1}{2 E} e^{i \lambda_{2} \theta_{12}}\left[\begin{array}{ccc}
m_{1}^{2} & 0 & 0 \\
0 & m_{2}^{2} & 0 \\
0 & 0 & m_{3}^{2}
\end{array}\right] e^{-i \lambda_{2} \theta_{12}}+a(x)\left[\begin{array}{ccc}
c_{13}^{2} & 0 & c_{13} s_{13} \\
0 & 0 & 0 \\
c_{13} s_{13} & 0 & s_{13}^{2}
\end{array}\right]\right\}\left[\begin{array}{c}
\tilde{\nu}_{e} \\
\tilde{\nu_{\mu}} \\
\tilde{\nu_{\tau}}
\end{array}\right]
$$

The CP phase $\delta$ disappears from the equation. It is due to the specific way that the matter effect comes in; $a(x)$ only appears in (1.1) element in the Hamiltonian matrix and therefore the matter matrix $\operatorname{diag}(a, 0,0)$ is invariant under rotation in $2-3$ space by $e^{i \lambda_{1} \theta_{23}}$. Then the rotation by the phase matrix $\Gamma$ does nothing.

It is clear from (8) that any transition amplitudes computed with $\tilde{\nu}_{\alpha}$ basis is independent of the $\mathrm{CP}$ violating phase. Of course, it does not immediately imply that the $\mathrm{CP}$ violating phase $\delta$ disappears in the physical transition amplitude $\left\langle\nu_{\beta} \mid \nu_{\alpha}\right\rangle$. The latter is related with the transition amplitude defined with $\tilde{\nu}_{\alpha}$ basis as

$$
\left\langle\nu_{\beta} \mid \nu_{\alpha}\right\rangle=T_{\alpha \gamma} T_{\beta \delta}^{*}\left\langle\tilde{\nu_{\delta}} \mid \tilde{\nu_{\gamma}}\right\rangle
$$

where $T$ is defined in (8) and its explicit form in our prametrization (5) of the mixing matrix reads

$$
T=\left[\begin{array}{ccc}
c_{13} & 0 & s_{13} \\
-s_{23} s_{13} e^{i \delta} & c_{23} & s_{23} c_{13} e^{i \delta} \\
-c_{23} s_{13} e^{i \delta} & -s_{23} & c_{23} c_{13} e^{i \delta}
\end{array}\right]
$$

It is then evident that the $\nu_{e}$ survival amplitude $\left\langle\nu_{e} \mid \nu_{e}\right\rangle$, and hence the probability, does not contain the $\mathrm{CP}$ violating phase:

$$
\left\langle\nu_{e} \mid \nu_{e}\right\rangle=c_{13}^{2}\left\langle\tilde{\nu_{e}} \mid \tilde{\nu_{e}}\right\rangle+s_{13}^{2}\left\langle\tilde{\nu_{\tau}} \mid \tilde{\nu_{\tau}}\right\rangle+c_{13} s_{13}\left(\left\langle\tilde{\nu_{e}} \mid \tilde{\nu_{\tau}}\right\rangle+\left\langle\tilde{\nu_{\tau}} \mid \tilde{\nu_{e}}\right\rangle\right)
$$

We have checked that this conclusion is not specific to the particular parametrization of the MNS matrix, as it should not.

Notice that the same statement does not apply to the $\nu_{\mu}$ disappearance amplitude:

$$
\begin{aligned}
\left\langle\nu_{\mu} \mid \nu_{\mu}\right\rangle= & s_{23}^{2} s_{13}^{2}\left\langle\tilde{\nu_{e}} \mid \tilde{\nu_{e}}\right\rangle+c_{23}^{2}\left\langle\tilde{\nu_{\mu}} \mid \tilde{\nu_{\mu}}\right\rangle+s_{23}^{2} c_{13}^{2}\left(\left\langle\tilde{\nu_{\tau}} \mid \tilde{\nu_{\tau}}\right\rangle\right. \\
& -c_{13} s_{23} s_{13}\left(e^{i \delta}\left\langle\tilde{\nu_{\mu}} \mid \tilde{\nu_{e}}\right\rangle+e^{-i \delta}\left\langle\tilde{\nu_{e}} \mid \tilde{\nu_{\mu}}\right\rangle\right)+c_{23} s_{13} c_{13}\left(e^{i \delta}\left\langle\tilde{\nu_{\mu}} \mid \tilde{\nu_{\tau}}\right\rangle+e^{-i \delta}\left\langle\tilde{\nu_{\tau}} \mid \tilde{\nu_{\mu}}\right\rangle\right) \\
& -s_{23}^{2} c_{13} s_{13}\left(\left\langle\tilde{\nu_{e}} \mid \tilde{\nu_{\tau}}\right\rangle+\left\langle\tilde{\nu_{\tau}} \mid \tilde{\nu_{e}}\right\rangle\right)
\end{aligned}
$$


One clearly sees the $\delta$ dependence in the survival amplitude of $\mu$ neutrinos in (12). Notices also that it's dependence is not necessarily $\cos \delta$ because

$$
\left\langle\tilde{\nu_{\beta}}(x) \mid \nu_{\alpha} \tilde{(}(0)\right\rangle \neq\left\langle\nu_{\alpha}(x) \mid \nu_{\beta}(0)\right\rangle
$$

owing to the fact that $a(0) \neq a(x)$ in general.

We now turn to the problem of $\mathrm{CP}$ violating effect due to the next-to-leading order correction of the standard electroweak interaction. It was noticed by Botella, Lim, and Marciano [16] that it gives rise to a small splitting between the indices of refraction of muon and tau neutrinos; the matter effect matrix in (4), diag.(a,0,0), becomes diag.(a,0,b) in an appropriate phase convention of the neutrino wave function. With a suitable redefinition of the Fermi constant $G_{F} a(x)$ is still given by the same form, $a(x)=\sqrt{2} G_{F} N_{e}(x)$, and the ratio $b / a$ is computed to be 16

$$
\begin{aligned}
\frac{b(x)}{a(x)} & =-\frac{3 \alpha}{2 \pi \sin ^{2} \theta_{W}}\left(\frac{m_{\tau}}{m_{W}}\right)^{2}\left[2 \ln \frac{m_{\tau}}{m_{W}}+\frac{5}{6}\right] \\
& \simeq 5.02 \times 10^{-5}
\end{aligned}
$$

for an isoscalar medium where $m_{\tau}$ and $m_{W}$ denote the masses of tau lepton and $\mathrm{W}$ boson, respectively. With the next-to-leading order effect the Hamiltonian matrix in the evolution equation (8) in $\tilde{\nu}$ basis has the additional term

$$
b(x)\left[\begin{array}{ccc}
c_{23}^{2} s_{13}^{2} & c_{23} s_{23} s_{13} e^{-i \delta} & -c_{23}^{2} c_{13} s_{13} \\
c_{23} s_{23} s_{13} e^{i \delta} & s_{23}^{2} & -c_{23} s_{23} c_{13} e^{i \delta} \\
-c_{23}^{2} c_{13} s_{13} & -c_{23} s_{23} c_{13} e^{-i \delta} & c_{23}^{2} c_{13}^{2}
\end{array}\right] .
$$

Clearly the $\tilde{\nu}$ evolution is affected by the CP violating phase. Its effect is however small, giving rise to the small correction term in the flavor conversion probability which is of the order of $b / a \simeq 5 \times 10^{-5}$.

If the neutrinos have hierarchy in their masses in such a way that the $\Delta m_{12}^{2}$ relevant for the solar neutrino conversion is much smaller than that of the atmospheric neutrino anomaly, i,e., $\Delta m_{13}^{2} \simeq \Delta m_{23}^{2} \equiv \Delta M^{2} \gg \Delta m_{12}^{2}$ then $\mathrm{CP}$ violation due to higher order effects are 
suppressed even further. To give a feeling we compute the next-to-leading order correction to the $\nu_{e}$ survival probability under the adiabatic approximation. We also restrict ourselves to the leading order perturbative correction of the effects of third generation neutrinos. We give here only the result, leaving the detail to ref. [17].

$$
\begin{aligned}
P\left(\nu_{e} \rightarrow \nu_{e}\right)= & c_{13}^{4}\left(c_{\omega}^{2} c_{12}^{2}+s_{\omega}^{2} s_{12}^{2}\right)+s_{13}^{4} \\
& +\frac{4 E}{\Delta M^{2}}\left(a-b c_{23}^{2}\right) c_{13}^{2} s_{13}^{2}\left[c_{13}^{2}\left(c_{\omega}^{2} c_{12}^{2}+s_{\omega}^{2} s_{12}^{2}\right)-s_{13}^{2}\right] \\
& +\frac{4 E}{\Delta M^{2}} b c_{13}^{2}\left(c_{12}^{2}-s_{12}^{2}\right) c_{\omega} s_{\omega} c_{23} s_{23} c_{13}^{2} s_{13} \cos \delta
\end{aligned}
$$

where $\Delta M^{2}=\Delta m_{23}^{2} \simeq \Delta m_{13}^{2}$ as defined above. The angle $\omega$ in (16) is the value of an angle at the solar core;

$$
\begin{aligned}
\sin 2 \omega & =\frac{B}{\sqrt{B^{2}+\frac{1}{4} D^{2}}} \\
B & =\left|\frac{\Delta m_{12}^{2}}{2 E} c_{12} s_{12}+b c_{23} s_{23} s_{13} e^{-i \delta}\right| \\
D & =\frac{\Delta m_{12}^{2}}{2 E}\left(c_{12}^{2}-s_{12}^{2}\right)-a c_{13}^{2}-b\left(c_{23}^{2} s_{13}^{2}-s_{23}^{2}\right)
\end{aligned}
$$

which parametrizes matter mass eigenstate in $2 \times 2$ submatrix. Notice that the $\mathrm{CP}$ violating effect comes out in a form very reminiscent of the Jarlskog factor, $J=c_{12} s_{12} c_{23} s_{23} c_{13}^{2} s_{13} \sin \delta$, apart from modification by the matter effect.

As clearly seen in (16), the CP violation effect is suppressed, in addition to the possible smallness of angle factors, by two small ratios,

$$
\frac{b}{\frac{\Delta M^{2}}{2 E}} \sim \frac{b}{a} \frac{\Delta m_{12}^{2}}{\Delta M^{2}} \simeq 5 \times\left(10^{-8}-10^{-7}\right)
$$

assuming that $\Delta m_{12}^{2}=10^{-6}-10^{-5} \mathrm{eV}^{2}$ and $\Delta M^{2}=10^{-3} \mathrm{eV}^{2}$.

We argue that the double suppression of the CP phase effect is not the artifact of the adiabatic approximation. Since $b$ carries the dimension of mass, it must be compensated by $\Delta m^{2} / E$. The $\Delta m^{2}$ has to be $\Delta M^{2}$ because the $\mathrm{CP}$ violation must vanish in an effective two-flavor limit $\Delta M^{2} \rightarrow \infty$. By the similar argument one can show that the $\mathrm{CP}$ violating term in $P\left(\nu_{\mu} \rightarrow \nu_{\mu}\right)$ obtains a mild suppression factor $\sim a E / \Delta M^{2} \simeq 0.1$ in the earth crust. 
In this paper we have proven that the effect of $\mathrm{CP}$ violating phase disappears from the survival probability $P\left(\nu_{e} \rightarrow \nu_{e}\right)$ within the framework of the standard model of electroweak interactions with minimal extension of including neutrino masses and mixing.

Several concluding remarks are in order:

(1) Unfortunately, our result implies that it is practically impossible to detect the effect of $\mathrm{CP}$ violating phase in solar neutrino measurements even with highest attainable accuracies.f (2) Our "no-go theorem" and hence the similar conclusion does apply to long-baseline electron (anti-) neutrino disappearance experiments. Muon (anti-) neutrino disappearance can be a hope but again we will face with the question of measurable CP odd observables.

(3) We emphasize that our result does not mean all negative; it implies that super highstatistics solar neutrino experiments in the future will provide a clean laboratory for precise determination of the lepton mixing angles without any uncertainties due to the leptonic Kobayashi-Maskawa phase.

(4) It is interesting to examine how our "theorem" would be invalidated by introduction of new neutrino properties which may exist in less conservative extention of the standard model.

The research of HM is partly supported by the Grant-in-Aid for Scientific Research in Priority Areas No. 11127213, and by the Grant-in-Aid for International Scientific Research No. 09045036, Inter-University Cooperative Research, Ministry of Education, Science, Sports and Culture.

$\dagger$ Of course, even if the effect turns out to be reasonablely large, we would have to worry about next on how to define the CP-violating observables to actually design the experiments. 


\section{REFERENCES}

[1] T. Kajita, Talk at 18th International Conference on Neutrino Physics and Astrophysics (Neutrino '98), June 4-9, 1998, Takayama, Japan, hep-ex/9810001, to appear in Proceedings; Y. Fukuda et al. (SuperKamiokande collaboration), Phys. Rev. Lett. 81 (1998) 1562.

[2] N. Cabbibo, Phys. Rev. Lett. 10 (1963) 531; M. Kobayashi and T. Maskawa, Prog. Theor. Phys. 49 (1973) 652.

[3] P. Ramond, Opening talk at 18th International Conference on Neutrino Physics and Astrophysics (Neutrino '98), June 4-9, 1998, Takayama, Japan, hep-ph/9809401, to appear in Proceedings.

[4] Z. Maki, M. Nakagawa and S. Sakata, Prog. Theor. Phys. 28 (1962) 247.

[5] M. Fukugita and T. Yanagida, Phys. Lett. B174 (1986) 45.

[6] N. Cabbibo, Phys. Lett. B72, (1978) 333; V. Barger, K. Wisnant and R. J. N. Phillips, Phys. Rev. Lett. 45 (1980) 2084; S. Pakvasa, in Proceedings of the XXth International Conference on High Energy Physics, edited by L. Durand and L. G. Pondrom, AIP Conf. Proc. No. 68 (AIP, New York, 1981), Vol. 2, pp. 1164.

[7] J. Arafune and J. Sato, Phys. Rev. D55 (1997) 1653; J. Arafune, M. Koike and J. Sato, Phys. Rev. D56 (1997) 3093.

[8] H. Minakata and H. Nunokawa, Phys. Lett. B413 (1997) 369; Phys. Rev. D57 (1998) 4403.

[9] M. Tanimoto, Phys. Rev. D55 (1997) 322; Prog. Theor. Phys. 97 (1997) 901.

[10] S. M. Bilenky, C. Giunti and W. Grimus, Phys. Rev. D58 (1998) 033001.

[11] A. De Rujula, M. B. Gavela, and P. Hernandez, Nucl. Phys. B547 (1999) 21.

[12] C. Jarlskog, Phys. Rev. Lett. 55 (1985) 1039. 
[13] L. Wolfenstein, Phys. Rev. D17 (1978) 2369.

[14] L.-L. Chau and W.-Y. Keung, Phys. Rev. Lett. 53 (1984) 1802; T. K. Kuo and J. Pantaleone, Phys. Lett. B198 (1987) 406; S. Toshev, Phys. Lett. B226 (1989) 335.

[15] T. K. Kuo and J. Pantaleone, Phys. Rev. D35 (1987) 3432.

[16] F. J. Botella, C.-S. Lim, and W. J. Marciano, Phys. Rev. D35 (1987) 896.

[17] S. Watanabe and H. Minakata, in preparation. 